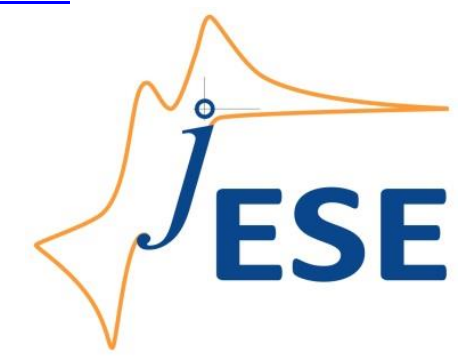

Open Access : : ISSN 1847-9286

www.jESE-online.org

Original scientific paper

\title{
Solvent dielectric effect on electrochemical properties of 3,4-propylenedioxythiophene
}

\author{
Keziban Huner ${ }^{1,2}$, Abdulkadir Sezai Sarac ${ }^{1,3, 凶}$ \\ ${ }^{1}$ Department of Chemistry, Istanbul Technical University,Maslak, 34469 Istanbul, Turkey \\ ${ }^{2}$ Department of Chemistry, Yildiz Technical University, Esenler, 34220 Istanbul, Turkey \\ ${ }^{3}$ Polymer Science and Technology, Istanbul Technical University, Maslak, 34469 Istanbul, Turkey \\ Corresponding author: ${ }^{\bowtie}$ sarac@itu.edu.tr
}

Received: June 28, 2021; Accepted: September 13, 2021; Published: October 1, 2021

\begin{abstract}
The present study is focused on the electrochemical properties of poly(3,4-propylenedioxythiophene) (Poly(ProDOT)), electrocoated on the single carbon-fiber microelectrode (SCFME) in different electrolytic media, with different solvent dielectric constants (35.9, 41.7, 47.5, 53.3, 59.1 and 64.9). The highest deposition charge density of $24.49 \mathrm{mC} \mathrm{cm}{ }^{-2}$ and the highest specific capacitance of $23.17 \mathrm{mF} \mathrm{cm} \mathrm{cm}^{-2}$ were obtained for Poly(ProDOT) synthesized in a medium with the lowest solvent dielectric constant ( $\varepsilon=35.9)$. Electrochemical impedance spectroscopy (EIS) results of Poly(ProDOT) coated SCFME measured at open circuit potential showed continuously increased impedance magnitudes as $\varepsilon$ was increased from 35.9 to 59.1. For all films, almost capacitive impedance responses at lower frequencies at least were obtained. The highest capacitance was observed for the polymer film synthesized in the medium of $\varepsilon=35.9$. The impedance of this film was also measured in different solvent mixtures with different dielectric constants at open circuit potential.
\end{abstract}

\section{Keywords}

carbon fiber; surface modification; electropolymerization; polythiophene derivate; electrochemical impedance spectra.

\section{Introduction}

Today, electrochromic, optical and thermoelectric properties of conductive polymers used in many fields continue to be investigated [1-5]. The conductive polymer materials with enhanced properties can be produced by electrochemical methods [6-8]. Electrochemical properties of electrochemically synthesized conductive polymers can be investigated by cyclic voltammetry (CV) and electrochemical impedance spectroscopy (EIS) [9-12]. Electrochemical impedance spectroscopy can be used both for volume and interfacial region studies and is associated with time constants ranging from minutes to microseconds [13]. 
In conducting polymers of importance for biomedical devices, low interfacial impedance and enhanced charge storage capacity are generally considered essential. It was already shown that electroplating with poly(3,4-alkylenedioxythiophene) reduced electrical impedance in biomedical devices and produced films with sufficient softness and large surface area [14-16]. Due to these film properties, there is a significant reduction of the electrical impedance of biomedical devices in the biologically relevant frequency range (around $1000 \mathrm{~Hz}$ ), and just this frequency corresponds to the typical pulse width of a neural signal (around 1-2 ms) [17]. Therefore, poly(3,4-alkylenedioxythiophene) and its derivatives are promising electronic materials used in organic bioelectronics for biosensing [18-21]. Carbon-based electrodes electrochemically coated with Poly(ProDOT) exhibit enhanced capacitive behavior and are promising materials for supercapacitor applications [22-24].

Micron size carbon fibers having low resistivity, high surface area, and small crystallites formed in the stacking direction during electropolymerization can improve the interface properties between polymer and carbon fiber. The approach of using a single carbon-fiber rather than a fiber bundle has the advantage of precise characterization of the coated film by electrochemical techniques. Conductive polymers electrocoated on CFMEs were previously studied and reported in detail by Sarac and coworkers [25-27]. FTIR-ATR, AFM and SEM characterizations of Poly(ProDOT) and Poly(ProDOT-co$\mathrm{N}$-phenylsulfonyl pyrrole) films synthesized by electropolymerization in $0.1 \mathrm{M} \mathrm{NaClO} / \mathrm{MeCN}$ were previously studied by the same group, and some characterization details are reported in [28].

In this study, polymer of 3,4-propylenedioxythiophene (ProDOT), a derivative of polythiophene (PTH) [29-31] was electro-coated specifically on a single carbon-fiber microelectrode (SCFME) in different electrolytic solutions having different solvent dielectric constants. The effect of the dielectric constant of the solvent on electrochemical properties of Poly(ProDOT) coated SFCME was investigated in detail by CV and EIS techniques.

\section{Experimental}

\section{Chemicals}

3,4-propylenedioxythiophene (ProDOT), propylene carbonate (PC), and tetrabutylammonium hexafluorophosphate (TBAPF6) were purchased from Aldrich. Acetonitrile (MeCN) and propylene carbonate (PC) were obtained from Riedel de Haën. Carbon fibers of Aksa Acrylic Co. were used in all experiments. All chemicals were of high purity.

\section{Electrochemical experiments}

Electrochemical polymerization was performed potentiodynamically by CV technique using a potentiostat 2263 Electrochemical Analyser (Princeton Applied Research, USA) interfaced to a PC and controlled by PowerSuit software package in one-compartment three-electrode cell. The electrochemical behavior of polymer samples prepared in different solutions was examined by the CV technique with the same three-electrode system. SCFME with an area of $\sim 2.19 \times 10^{-3} \mathrm{~cm}^{2}$, a platinum wire, and a silver wire were used as working, counter and reference electrodes, respectively. Ag wire used as the reference electrode was checked and calibrated by ferrocene $(0.1 \mathrm{M})$.

EIS measurements of Poly(ProDOT) coated SFCME in different dielectric media were performed using potentiostat 2263 Electrochemical Analyser (Princeton Applied Research, USA) within a frequency range between $100 \mathrm{kHz}$ and $0.01 \mathrm{~Hz}$ and $A C$ signal amplitude of $10 \mathrm{mV}$, at applied potentials of $-0.2,0.0$, and $0.2 \mathrm{~V}$ and open circuit potential (OCP) condition. Electrochemical impedance software (PowerSine) was used to carry out impedance measurements of polymer coated SCFMEs in monomer-free electrolytic solution with the same three-electrode system. 
Impedance data were analyzed using ZSimpWin (Version 3.1) AC impedance data analysis software (Princeton Applied Research).

\section{Results and discussion}

\section{Electropolymerization}

Poly(ProDOT) was obtained from $2 \mathrm{mM}$ ProDOT monomer solution by performing $20 \mathrm{CV}$ cycles at the scan rate of $50 \mathrm{mV} / \mathrm{s}$ in the potential range of $-0.5-1.5 \mathrm{~V}$, and the recorded graphical results were compared. In all experiments, $0.1 \mathrm{M} \mathrm{TBAPF}_{6}$ was used as the supporting electrolyte, while acetonitrile (MeCN), propylene carbonate (PC), and their mixtures were used as solvents. Equation (1) was used to calculate dielectric constant values of solvent mixtures:

$$
\varepsilon_{\mathrm{m}}=\varepsilon_{1} X_{1}+\varepsilon_{2} X_{2}
$$

The dielectric constant of solvent 1 is $\varepsilon_{1}$, the volume fraction of the same solvent is $x_{1}, \varepsilon_{2}$ is the dielectric constant of solvent 2 , and the volume fraction of solvent 2 is $x_{2}$ [32]. Dielectric constants of the solvents and their mixtures at room temperature are given in Table 1.

Table 1. Dielectric constants of solvents and their mixtures (at $293.15 \mathrm{~K}$ ).

\begin{tabular}{ccc}
\hline Solvent & Solvent ratio $(\mathrm{V} / \mathrm{V})$ & Dielectric constant \\
\hline MeCN & 1 & 35.9 \\
\hline MeCN-PC & $4: 1$ & 41.7 \\
\hline MeCN-PC & $3: 2$ & 47.5 \\
\hline MeCN-PC & $2: 3$ & 53.3 \\
\hline MeCN-PC & $1: 4$ & 59.1 \\
\hline PC & 1 & 64.9
\end{tabular}

Electropolymerization of ProDOT in electrolytic solutions with different solvent dielectric constants is shown in Figure 1. As the number of cycles increased, the oxidation-reduction current values increased, mostly for the coating obtained at $\varepsilon=35.9$ (Figure 1a). Coatings obtained at solvent dielectric constants 41.7, 47.5 and 64.9 changed to a much lesser extent (Figure $1 \mathrm{~b}-\mathrm{d}$ ), while these obtained at the solvent dielectric constants of 53.3 and 59.1 did not change at all (not shown). As the solvent dielectric constant increased from 35.9 to 59.1, the maximum oxidation current value decreased from 3.1 to $0.003 \mu \mathrm{A}$ and then increased to $0.2 \mu \mathrm{A}$ in the electrolytic solution with a solvent dielectric constant of 64.9. The inner graphs in Figure 1 show the oxidation-reduction current values versus scanning rate in electrolytic solutions where coatings were made but without monomer. Accordingly, the current values increased as the scan rate increased from 50 to $200 \mathrm{mV} \mathrm{s}^{-1}$, except in electrolytic solutions with solvent dielectric constant of 53.3 and 59.1. It can be said that the system is diffusion-controlled $[27,33,34]$ since the current values of the electrocoating obtained in electrolytic solutions with solvent dielectric constants of 35.9, 41.7, 47.5 and 64.9 increase with the increase of the scan rate.

The linear dependence of the peak current with the square root of scan rate occurs with electrodes dependent on the diffusion limited redox reaction, while for reaction molecules that adsorb on the electrode surface, peak current will be linearly dependent on the scan rate [35].

The graphs of anodic and cathodic currents against the scan rate and square root of the scan rate for $\varepsilon=35.9$ are given in Figure 2. The linear dependence of the peak current with the scan rate for Poly(ProDOT) coated SCFME in monomer-free solution indicates that redox reaction is primarily dependent on the adsorption of molecules. Note that for the diffusion limited redox reaction, the linear dependence of the peak current on the square root of scan rate was predicted [33,34]. 

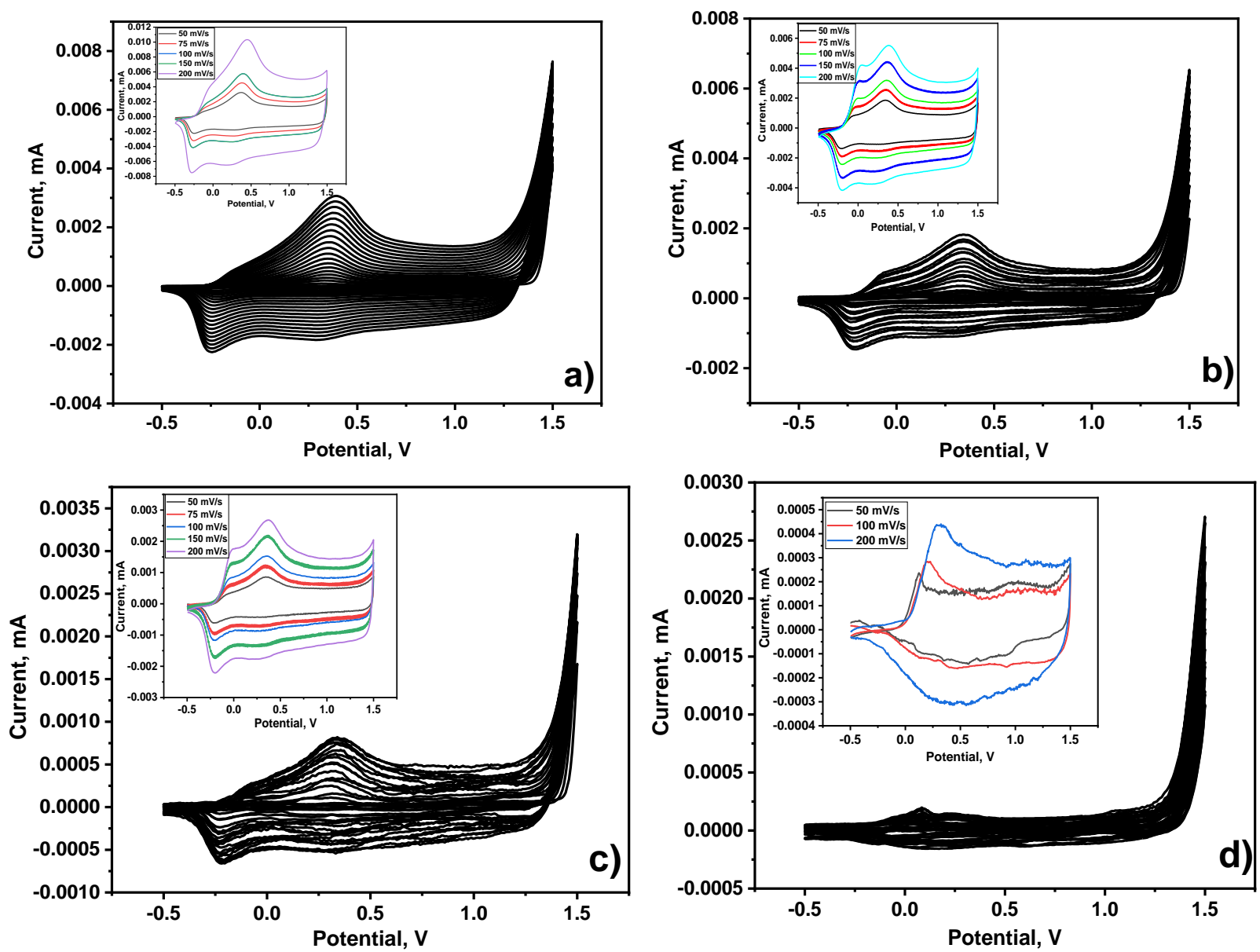

Figure 1. Cyclic voltammograms of polymerization (20 cycles, $50 \mathrm{mV} / \mathrm{s}$ ) at SCFME from $2 \mathrm{mM}$ ProDOT monomer in $0.1 \mathrm{M} \mathrm{TBAPF}_{6}$ and solvent dielectric constant: a) 35.9; b) 41.7; c) 47.5; d) 64.9. Inset graphs: CVs of Poly(ProDOT) in monomer-free $0.1 \mathrm{M} \mathrm{TBAPF}_{6}$ at different scan rates $(50-200 \mathrm{mV} / \mathrm{s})$. Surface area of $W E \sim 2.19 \times 10^{-3} \mathrm{~cm}^{2}$
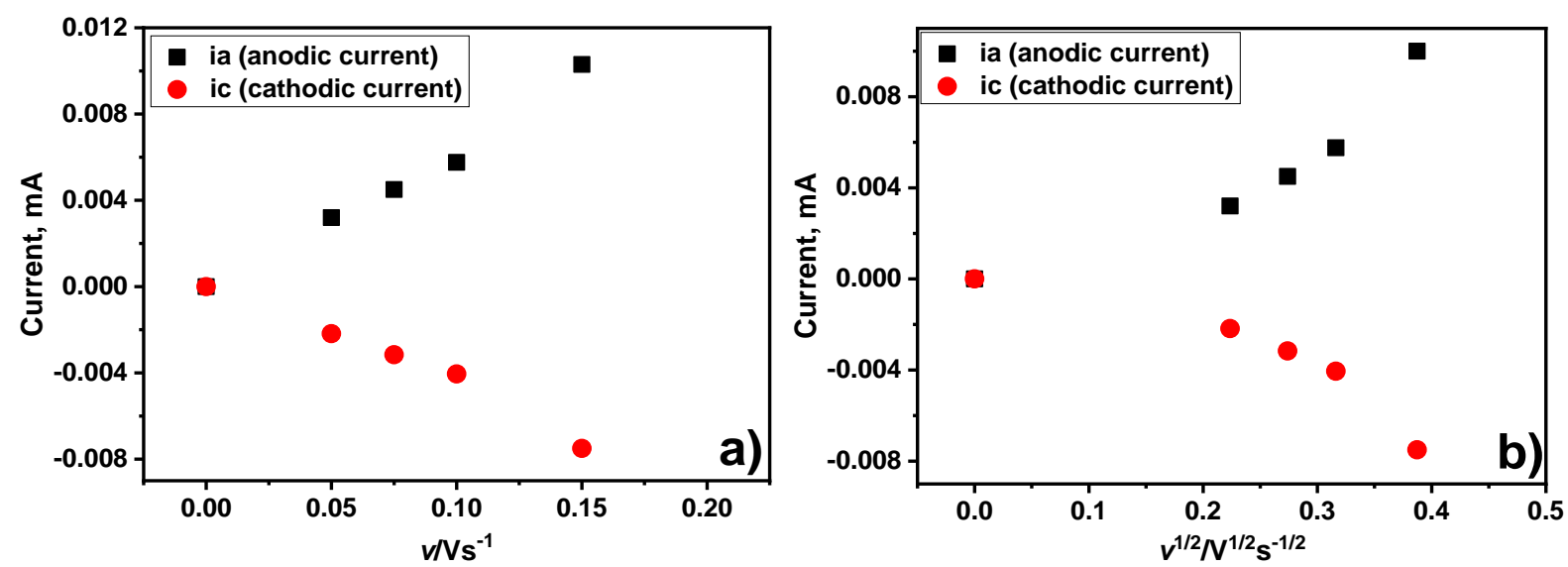

Figure 2. Plot of anodic ( $\left.\mathrm{i}_{a}\right)$ and corresponding cathodic ( $\left.\mathrm{i}_{c}\right)$ peak current vs. a) scan rate; b) square root of scan rate for $\varepsilon=35.9$ (inset of Fig. 1a)

The graph of anodic and cathodic peak potential values against the scan rate is given in Figure 3 . With increasing scan rate, the anodic peak potential is shifted positively, while the cathodic peak potential is shifted negatively. An increase of the scan rate can limit the time interval of the electrochemical process, which results in a shift towards more positive potentials (for anodic peaks) and negative potentials (for cathodic peaks). The shift is mainly due to the delay of the electro- 
chemical reaction due to the shortness of time compared to a lower scan rate that allows more time for the reaction to occur (Figure 3).

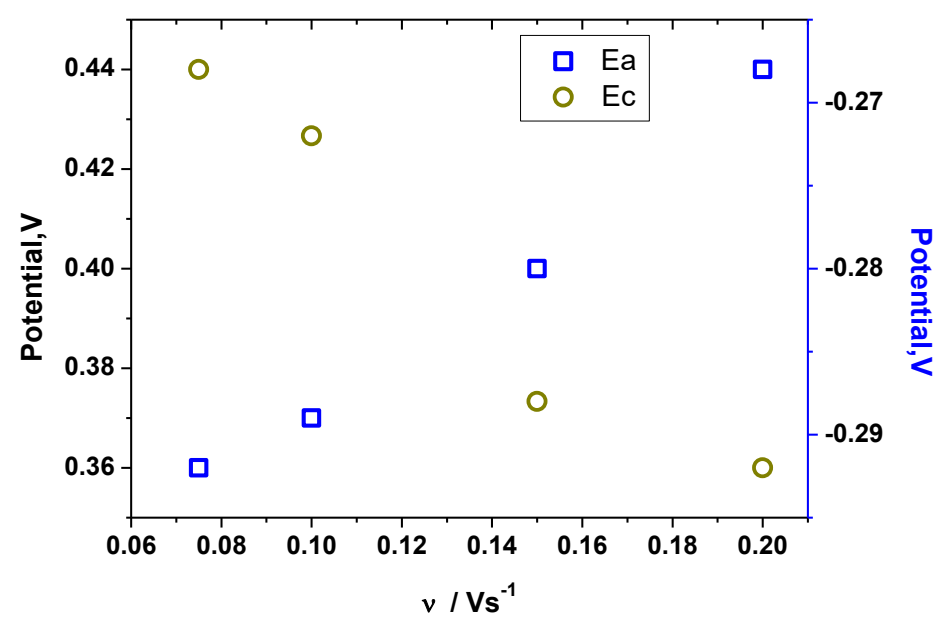

Figure 3. Anodic $\left(\mathrm{E}_{a}\right)$ and cathodic $\left(\mathrm{E}_{c}\right)$ peak potential values vs. scan rate for $\varepsilon=35.9$ (inset of Fig. 1a)

Figure 4 presents schematic steps of the electrochemical polymerization of ProDOT on the SCFME. Electron transfer from ProDOT monomer to SCFME working electrode due to oxidation is the first step of electropolymerization. In the next step, coupling reactions with the formed ProDOT radical cations, followed by a dehydrogenation step can be realized. By coupling radical cations, oligomers are formed, and further oxidation of these oligomeric and polymeric radical cations can interact with electrolyte anions $\left(\mathrm{PF}_{6}{ }^{-}\right)$, depending on the solvent medium dielectricity.

Solvent with a high dielectric constant is easily polarized. Polarization allows countercharges to be placed around an ion, which results in coulombic interactions between solvent and radical cations of Poly(ProDOT), and reduces the polymerization ability of oligomers of ProDOT during the radical cation coupling (Figure 4). Therefore, the deposition charge density of polymer ( $\alpha$ thickness) was found to decrease by increasing the dielectric constant of the medium.

Solvents influence the solubility, stability and reaction rates, and choosing the appropriate solvent allows thermodynamic and kinetic control over a chemical reaction. Stabilization of the intermediates may occur through different non-covalent interactions with the solvent i.e., H-bonding, dipole-dipole interactions, van der Waals interactions, etc. Stabilization of radical cations (ProDOT radical cation and/or oligomeric radical cation) is larger in polar solvents than in less polar solvents. Polar solvent one with a high dielectric constant - will stabilize radical cations by forming interactions and reducing the reactivity of these intermediates with each other (Figure 4).

The deposition charge density of the polymer electrogrowth $(q)$ and specific capacitance $\left(C_{\mathrm{sp}}\right)$ in monomer free solution were calculated from CV measurements according to equations (2) and (3):

$$
\begin{gathered}
q=\frac{\int i \mathrm{~d} V}{2 v A} \\
C_{\mathrm{sp}}=\frac{q}{\mathrm{~d} V}
\end{gathered}
$$

where $\int i d V$ is the integrated area under the CV curve, $v$ is the scan rate, $V s^{-1} ; \Delta V / V$ is the potential window in $\mathrm{CV}$ measurements and $A / \mathrm{cm}^{2}$ is the electrode surface area. 


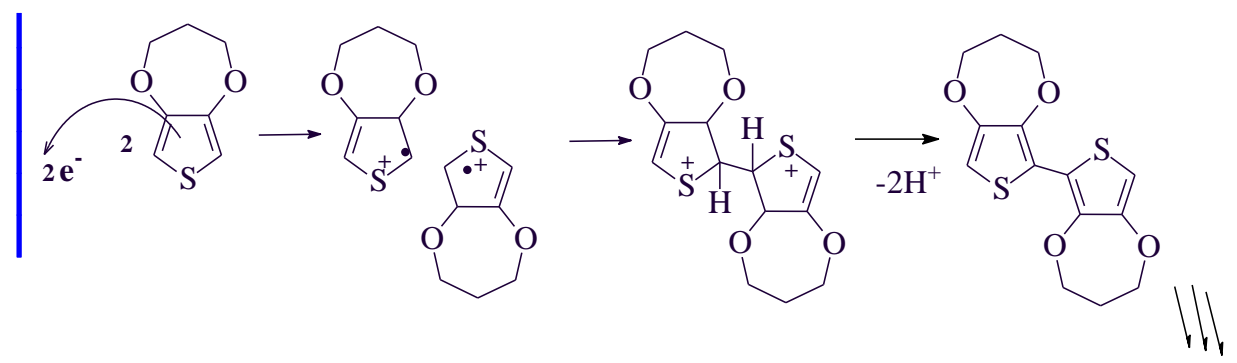

a
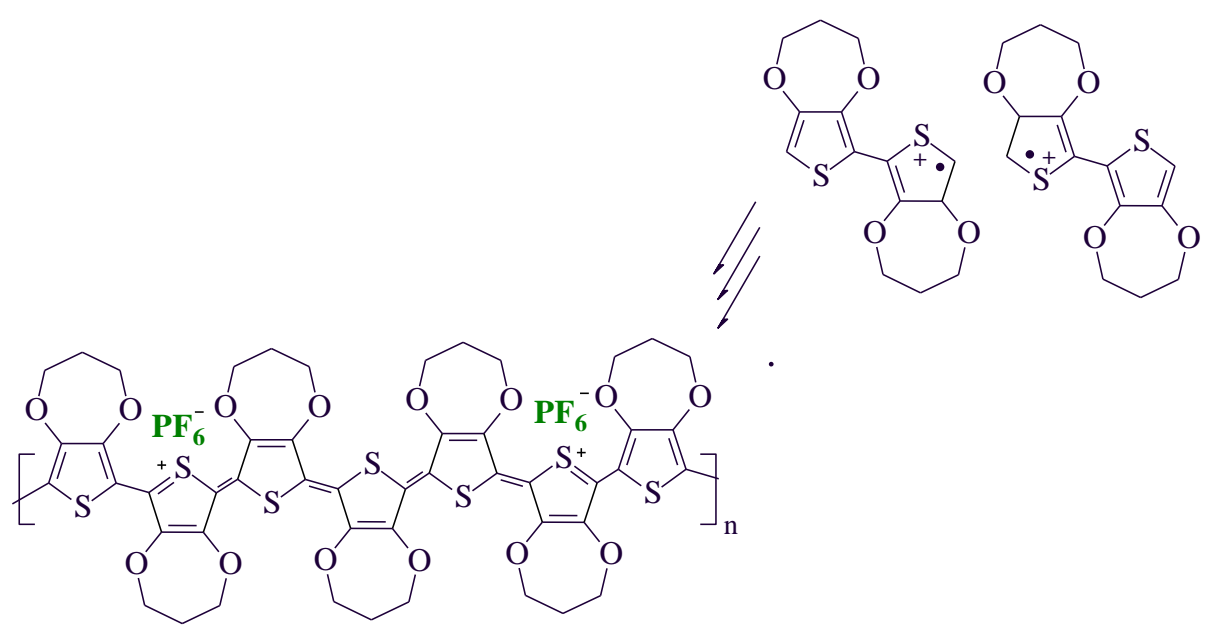

$\delta_{-}$

$\mathrm{CH}_{3} \mathrm{C} \equiv \mathrm{N}$

b

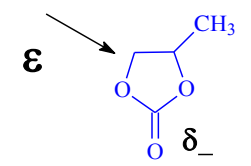

Figure 4. a) Tentative mechanism of Poly(ProDOT) electrodeposition onto SCFME and doping by anion of electrolyte $\left(\mathrm{PF}_{6}^{-}\right)$; b) change of dielectric media from low to high polarity

The graph of the calculated deposition charge density of ProDOT and specific capacitance (Eqs. 2 and 3 ) versus dielectric constant of the solvent, obtained from CVs of Poly(ProDOT) in monomerfree solution is shown in Figure 5.

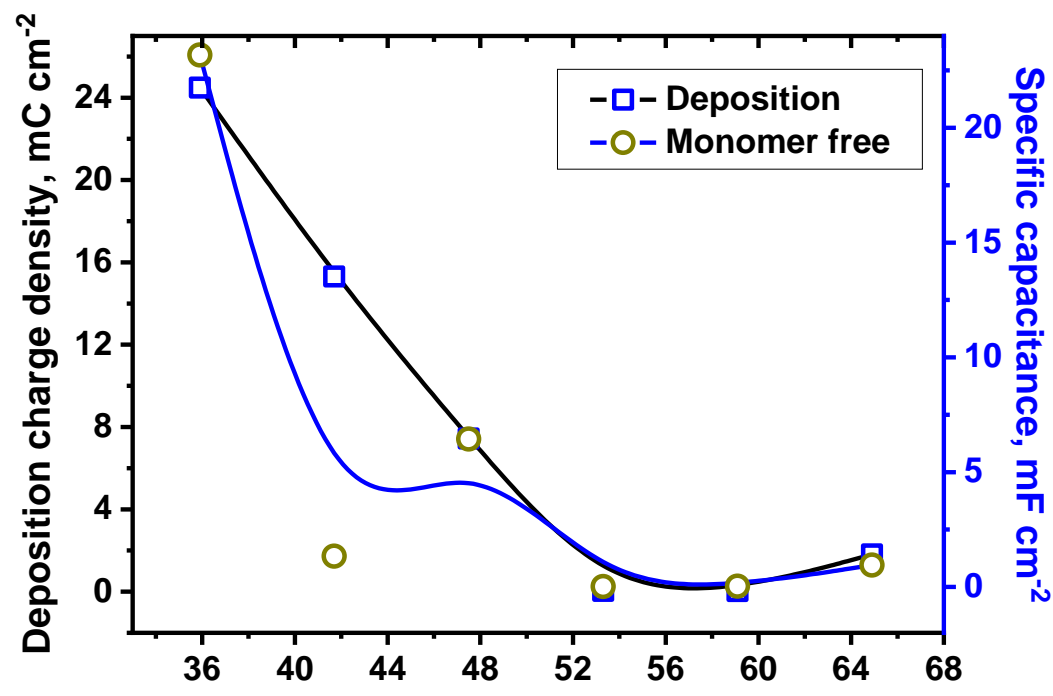

Dielectric constant

Figure 5. Deposition charge density of electrogrowth $(50 \mathrm{mV} / \mathrm{s}, 20$ cycles) of ProDOT (left axis), and specific capacitance (right axis) vs. dielectric constant of solvent obtained from CV $(200 \mathrm{mV} / \mathrm{s})$ of Poly(ProDOT) in monomer-free $0.1 \mathrm{MTBAPF_{6 }}$. Surface area of WE $\sim 2.19 \times 10^{-3} \mathrm{~cm}^{2}$ 
As the solvent dielectric constant increased, the deposition charge density in the system decreased. Accordingly, the highest deposition charge density value was obtained from the coating realized in the media with a dielectric constant of 35.9.

The deposition charge density of film electrogrowth can be generally correlated with its thickness which becomes lower at higher dielectric constants. According to Figure 5, specific capacitance values of these films show a similar trend with the exception of $\varepsilon=41.7$. The highest deposition charge density of $24.49 \mathrm{mC} \mathrm{cm}^{-2}$, and the highest specific capacitance of $23.17 \mathrm{mF} \mathrm{cm}^{-2}$, were obtained for Poly(ProDOT) synthesized in the solvent with the lowest dielectric constant $(\varepsilon=35.9)$.

\section{Electrochemical impedance spectroscopy}

Impedance $(Z)$ defined by Eq. (4) is the angular frequency $(\omega)$ dependent resistance of a system to the alternating current (AC) flow caused by the application of frequency-dependent potential. In the limit of zero frequency, impedance becomes frequency independent resistance against the electrical current, or DC resistance [36].

$$
Z=\frac{E(\omega)}{I(\omega)}
$$

Solution resistance is an important factor in electrochemical impedance measurements of an electrochemical cell. In a three-electrode system, the uncompensated solution resistance is measured between the working and the reference electrode [37].

EIS results are usually presented by plotting the measured impedance against the frequency $(f=\omega / 2 \pi)$. Impedance spectrum presentation is the frequency $(f)$ dependence of two real quantities (absolute impedance value $-|Z|$ and phase angle $-\varphi$ ). In the spectrum set as Bode plot, log $|Z|$ and $\varphi$ are drawn against log $\omega$. In Nyquist plot, the imaginary part of impedance $\left(Z_{i m}\right)$ is put against the real part of impedance $\left(Z_{\mathrm{re}}\right)$. For capacitive systems, complex capacitance, $C=(i \omega Z)^{-1}$, is the most valuable presentation, where total capacitance can be evaluated directly from a spectrum as $C_{\text {re }}$ for $\omega \rightarrow 0$. The impedance spectrum is generally studied with equivalent circuit modelling (ECM) containing different electric elements. Each element in ECM and its connection with other elements point to some specific physical event. There are three basic elements used in ECM [38-40]. The first is a resistor that appears in the Bode plot as a plateau having zero phase angle, a capacitor that appears as a straight line with a slope of -1 and phase angle of $-90^{\circ}$, and element related to the diffusion of reaction species, showing a straight line with a slope of -0.5 and phase angle of $-45^{\circ}$. In the Nyquist plot, resistance appears as a point at $Z_{\text {re, }}$ and capacitance as a vertical line (angle of $90^{\circ}$ ), where capacity value can be calculated from $Z_{\text {im }}$ value at certain $\omega$ as $C=\left(\omega Z_{i m}\right)^{-1}$. The element related to diffusion (Warburg element) shows a straight line having a slope of $45^{\circ}$.

EIS results of Poly(ProDOT) coated SCFMEs measured in monomer-free electrolytic solutions under open-circuit potentials are given in Figure 6. For each Poly(ProDOT) coated SCFME, EIS was taken in the same electrolytic solution from which the coating was made but under monomer-free conditions.

According to the EIS results in Figure 6a, for higher dielectric constants (53.3 and 59.1), more or less inclined capacitive lines are generally observed, with impedance much higher than these measured for low dielectric media $(35.9,41.7,47.5)$. Impedance for $\varepsilon=64.9$ lies somewhere between these two groups. Also, for higher $\varepsilon$ (53.3 and 59.1), it seems that Nyquist plots are started to bend with a significant increase of the real resistance at the lowest frequencies, tending to form a semicircle.

For all films, Bode plots in Figure $6 \mathrm{~b}$ show resistive impedance at higher frequencies and almost capacitive impedance responses at lower frequencies. Two groups of impedance spectra can be generally distinguished, a group with lower impedances ( $\varepsilon=35.9,41.7$ and 47.5$)$ and consequently 
higher capacitances and a group with higher impedances $(\varepsilon=53.3$ and 59.1) and hence lower capacitances. The impedance spectrum for $\varepsilon=64.9$ lies somewhere between these two groups. Bode phase plots in Figure $6 \mathrm{~d}$ clearly show that almost pure capacitive impedance response, characterized by phase angle near $-90^{\circ}$, are obtained for $\varepsilon=35.9$, and $\varepsilon=53.3$ and 59.1. The other films, including that for $\varepsilon=64.9$, show more complex behavior, with clear resistive contribution(s) making peaks in phase angle responses at higher frequencies and almost capacitive impedance responses at lower frequencies. Anyhow, it is clear that going from films formed at higher dielectric constants ( $\varepsilon=53.3$ and 59.1), there is a continuous change from almost pure capacitive response showing lower capacitance, via complex impedance response involving some resistance contribution(s) $(\varepsilon=41.7,47.5,64.9)$, to pure capacitive impedance response showing higher capacitance $(\varepsilon=35.9)$ (Figure $6 \mathrm{c}$ ).
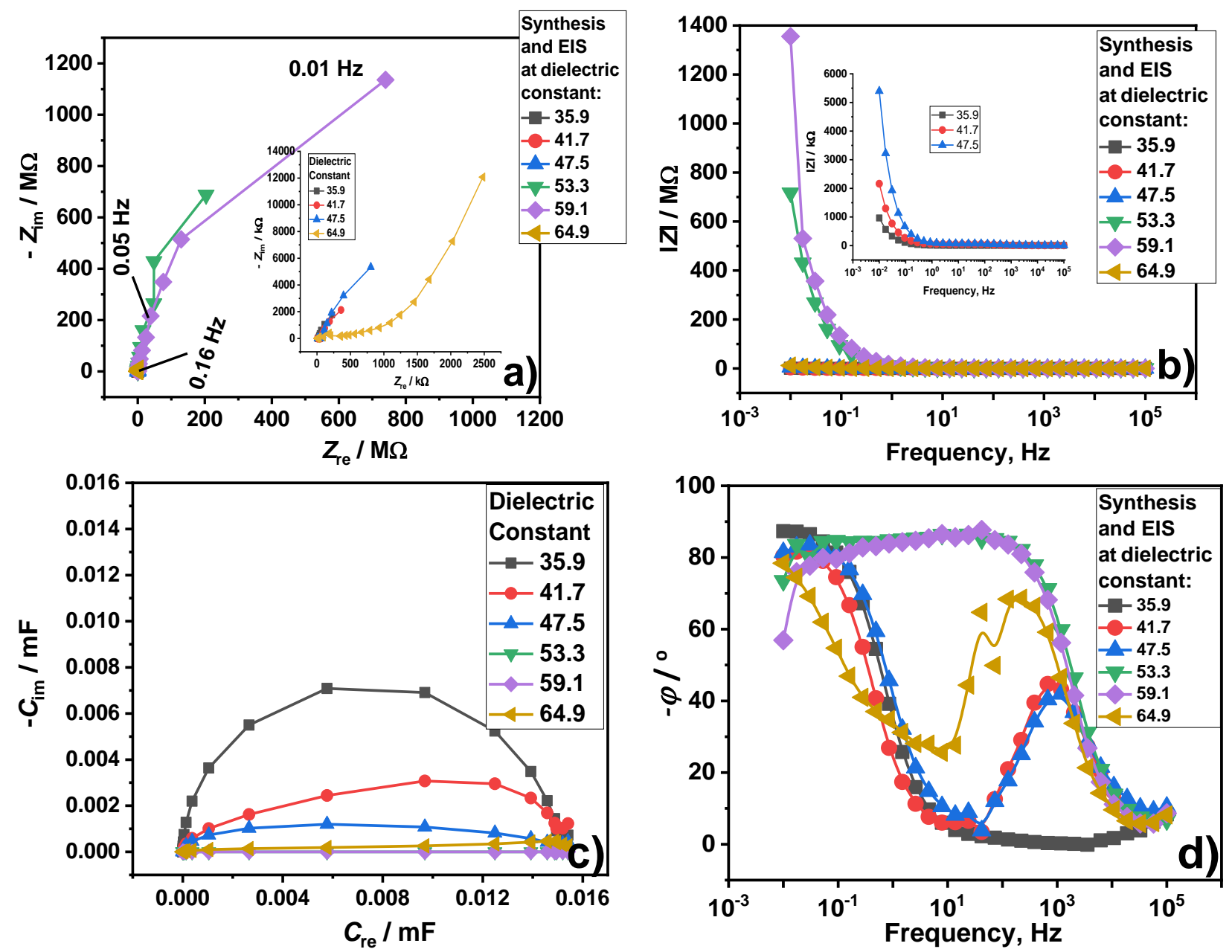

Figure 6. EIS results of Poly(ProDOT) synthesized in solvents with different dielectric constants and measured in monomer-free $0.1 \mathrm{M} \mathrm{TBAPF}_{6}$ under open circuit potential: a) Nyquist; b) Bode magnitude; c) complex capacitance; d) Bode phase plots

EIS results of Poly(ProDOT) coated SCFME, measured in monomer-free electrolytic solutions at different applied potentials (-0.2, 0.0 and $0.2 \mathrm{~V}$ ), are given in Figure 7. For each Poly(ProDOT) coated SCFME, EIS was taken in the same electrolytic solution from which the coating was made but without a monomer. Generally, films formed in solutions of higher dielectric constant ( $\varepsilon=53.3$ and 59.1) showed the highest and almost capacitive impedances, independent of the potential of measurements (Figure 7a-b). For other films at all three potential values, much lower impedances are generally observed. Phase angle Bode plots in Figure 7c show that almost pure capacitive impedance 
responses are observed for the films ( $\varepsilon=35.9,41.7$ and 47.5$)$, all measured at $0.2 \mathrm{~V}$. This might be related to the fact that $0.2 \mathrm{~V}$ is closest to the potential of redox peak of the faradic/doping process recorded by CVs shown in Figure 1. Other films measured at either 0.0 or $-0.2 \mathrm{~V}$, showed more complex behavior involving some clear resistance contribution(s), which is less prominent for films of lower $\varepsilon$ and the most prominent for the film $\varepsilon=64.9$ at both 0 and $-0.2 \mathrm{~V}$.

Generally, higher capacitances are obtained for polymer films formed in lower dielectric media than those from higher dielectric solvent media. In addition to the ordinary double-layer capacitance formed at interfacial regions of polymer films, high capacitance of conducting polymers originates from the film's active layer, where fast redox reaction with low charge transfer/transport resistances results in pseudocapacitance.
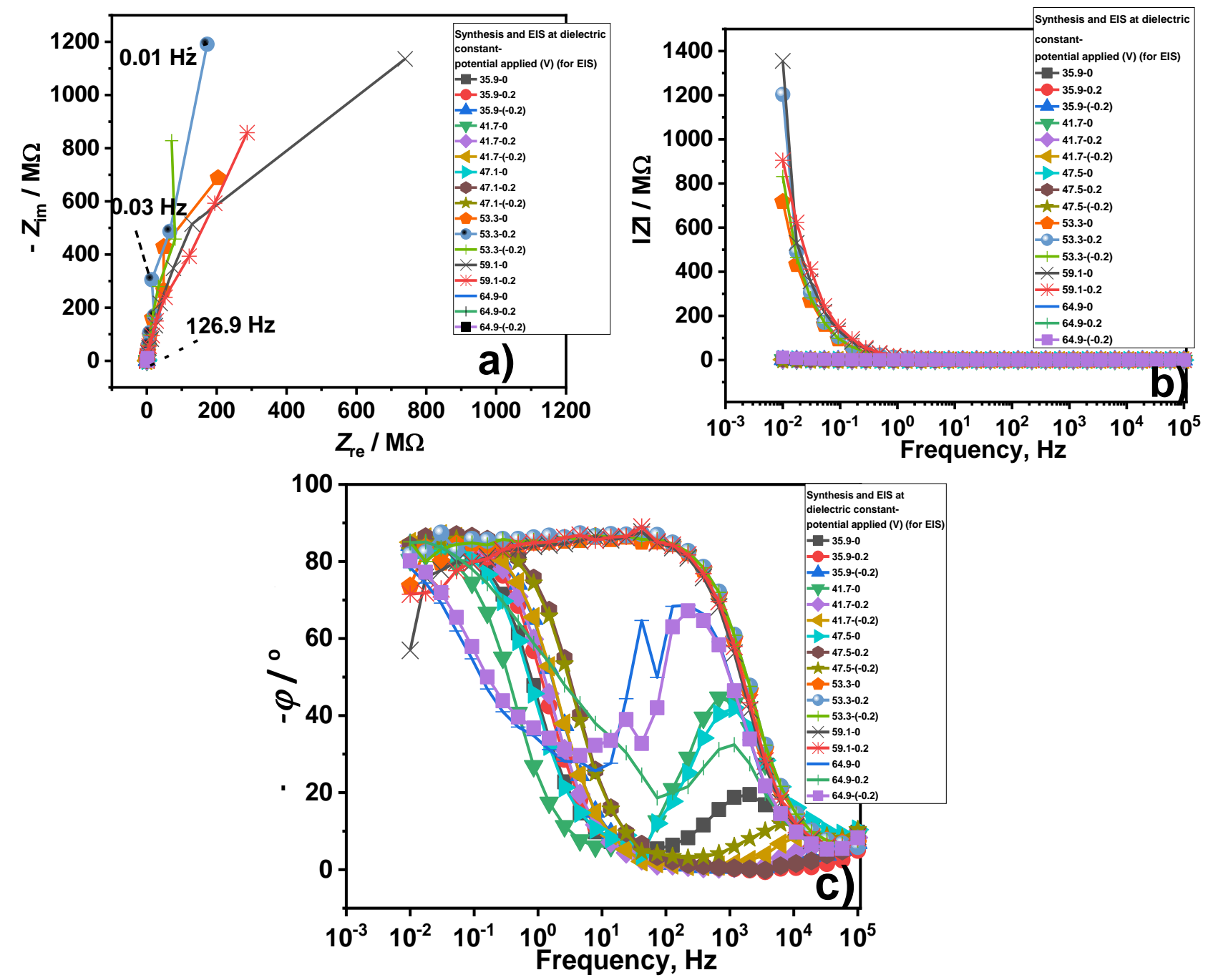

Figure 7. EIS results of Poly(ProDOT) synthesized in solvent mixtures with different dielectric constants and measured in monomer-free $0.1 \mathrm{MTBAPF} \mathrm{T}_{6}$ at $-0.2,0.0$ and $0.2 \mathrm{~V}$ : a) Nyquist;

b) Bode magnitude; c) Bode phase plots

EIS results of Poly(ProDOT) synthesized on SCFME in 0.1M TBAPF $6 / \mathrm{MeCN}, \varepsilon=35.9$ ) measured in different dielectric media under monomer free conditions at open circuit potential presented in Figure 8. Bode plots (Figure 8a and 8d), Nyquist plots (Figure 8b) and complex capacitance spectra (Figure $8 \mathrm{c}$ ) indicate that the polymer coating on the SCFME is very stable since all measured spectra are rather similar for different dielectric solvent media. 

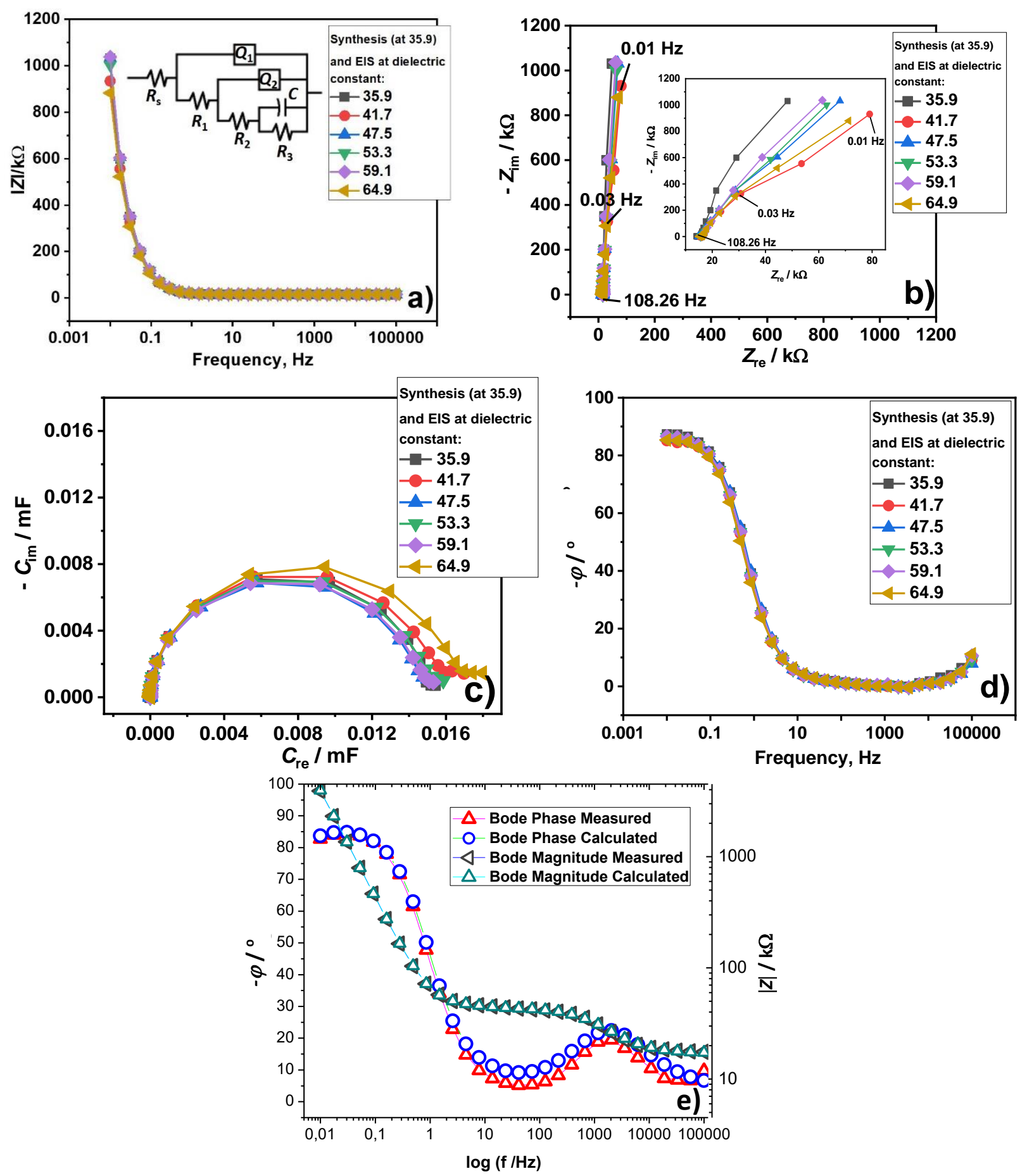

Figure 8. EIS results of Poly(ProDOT) synthesized in the solvent with $\varepsilon=35.9$ and measured in solvent mixtures with different dielectric constants at open circuit potential: a) Bode magnitude; b) Nyquist; c) complex capacitance; d) Bode phase plots; e) comparison of measured (Fig. $8 b$ and $8 d$ ) for $\varepsilon=35.9$, and calculated (according to ECM in Fig. 8a) Bode phase and magnitude plots

\section{Equivalent circuit modeling}

Poly(ProDOT) were synthesized by electrochemical polymerization in electrolytic solutions containing different solvent mixtures that have different dielectric constants. Electrochemical parameters of Poly(ProDOT) electrocoated on SCFME were evaluated with ZsimpWin program and complex nonlinear least squares (CNLS) analysis, using the presumed equivalent circuit model. If $\chi^{2}$ (Chi-squared) value obtained from the comparison between measured and calculated impedance spectrum is around $10^{-4}$, it means that the circuit fits the measured results. The value of $\chi^{2}$ is expressed as the sum of squares of the residuals. 
The model (ECM) drawn within Figure 8a was chosen for the electrical equivalent circuit modelling procedure. It consists of three resistances $\left(R_{\mathrm{s}}, R_{1}\right.$, and $\left.R_{2}\right)$, two CPE elements $\left(Q_{1}\right.$ and $\left.Q_{2}\right)$ and a pure capacitor $(C)$. The constant phase element (CPE) is used instead of ideal capacitors $(C)$ to compensate for the porosity, roughness, and inhomogeneity of the electrode surface in the system [41]. $R_{\mathrm{s}}$ should be ascribed to the pore, and uncompensated electrolyte resistances, a combination $\left(Q_{1}\right.$ and $\left.R_{1}\right)$ presents interfacial impedance, characterized by the double-layer capacitance $\left(C_{\mathrm{dl}}\right)$ and charge-transfer resistance $\left(R_{\mathrm{ct}}\right)$ in the first parallel combination [42]. The second parallel combination $\left(Q_{2}\right.$ and $\left.R_{2}+C\right)$ is related to the active interior of polymer film, where $R_{2}$ comprises ion transfer/transport resistances within the film of pseudocapacitance $(C) . R_{3}$ was put into ECM in Figure 8 a to account for not ideal capacitive impedance response at the lowest frequencies.

ECM was well fitted to the experimental data for Poly(ProDOT), as is already shown in Figure 8e by drawing measured and calculated impedance spectra for the film formed in the solvent with $\varepsilon=$ 39.5 and measured at the open circuit potential. Table 2 summarizes impedance parameter values obtained after fitting of ECM from Figure 8a to impedance spectra of Poly(ProDOT) obtained at some $\varepsilon$ and measured in monomer-free solution at $0.0 \mathrm{~V}$ (Figure 7 ).

Table 2. Values of elements calculated by ECM in Fig. 8a fitted to EIS results of Poly(ProDOT) presented in Fig. 7 for $0.0 \mathrm{~V}$. Surface area of $W E=2.19 \times 10^{-3} \mathrm{~cm}^{2}$

\begin{tabular}{|c|c|c|c|c|c|c|c|c|c|}
\hline$\varepsilon$ & $R_{\mathrm{s}} / \Omega \mathrm{cm}^{2}$ & $Q_{1} / \mu \mathrm{S} \mathrm{s}^{\mathrm{n}} \mathrm{cm}^{-2}$ & $n_{1}$ & $R_{1} / \Omega \mathrm{cm}^{2}$ & $Q_{2} / \mu \mathrm{S} \mathrm{s}^{\mathrm{n}} \mathrm{cm}^{-2}$ & $n_{2}$ & $R_{2} / \Omega \mathrm{cm}^{2}$ & $\mathrm{C} / \mathrm{F} \mathrm{cm}^{-2}$ & $R_{3} / \mathrm{k} \Omega^{2} \mathrm{~cm}^{2}$ \\
\hline 35.9 & 17.03 & 38.0 & 0.78 & 26.92 & 2610 & 0.96 & 26.25 & 0.01035 & 65.2 \\
\hline 41.7 & 15.96 & 11.3 & 0.89 & 113 & 2930 & 0.97 & $4.49 \times 10^{4}$ & -- & -- \\
\hline 47.5 & 20.64 & 11.2 & 0.85 & 136.8 & 1150 & 0.96 & $1.45 \times 10^{5}$ & -- & -- \\
\hline 59.1 & 23.76 & 4.65 & 0.97 & $3.73 \times 10^{5}$ & -- & -- & -- & -- & -- \\
\hline
\end{tabular}

Data in Table 2 show well resolved parameters for Poly(ProDOT) formed at $\varepsilon=35.9$. For higher $\varepsilon$, however, a reduced number of parameters was obtained, which is obviously due to higher resistance, primarily $R_{2}$ values. For polymer film formed at low $\varepsilon, R_{1}$ and $R_{2}$ resistances are relatively low, allowing a pseudocapacitance $(C)$ of about $10 \mathrm{mF} \mathrm{cm}{ }^{-2}$ to be achieved. $R_{1}$ and $R_{2}$ values for Poly(ProDOT) are significantly increased for higher $\varepsilon$, but at the same time, a decrease is observed for $Q$ values. Decrease of double layer capacitance with the increase of $\varepsilon$ of medium is due to doping of polymer cationic sites (Figure 4). $R_{1}$ and $R_{2}$ increase under higher dielectric constants, where the deposition charge densities decrease (Figure 5). This indicates that less thickness and less conductive films are obtained at a higher dielectric constant. Dielectric constant $(\varepsilon)$ is a measure of a substance's ability to insulate charges from each other. By considering solvent polarity, higher $\varepsilon$ means higher polarity and greater ability to stabilize charges. Increasing the polarity of the solvent increases the solvation of the anion of electrolyte $\left(\mathrm{PF}_{6}{ }^{-}\right)$. There will be an increase in dipole-dipole interactions between the solvent and dopant $\mathrm{PF}_{6}$. As the polarity of the solvent increases, the mobility of $\mathrm{PF}_{6}{ }^{-}$ions is reduced, reducing the polymer doping, which results in less conductive material than that formed at low dielectric conditions.

When prepared in favourable conditions of low solution dielectric constant, Poly(ProDOT) shows low resistance and high capacitance values (Figure 9). Due to these properties, Poly(ProDOT) can be used in biosensing applications because it has sufficient electronic conductivity and is also biocompatible and stable. Enzymes can be immobilized on conductive polymer coated electrodes in several ways, such as physical adsorption $[43,44]$. 


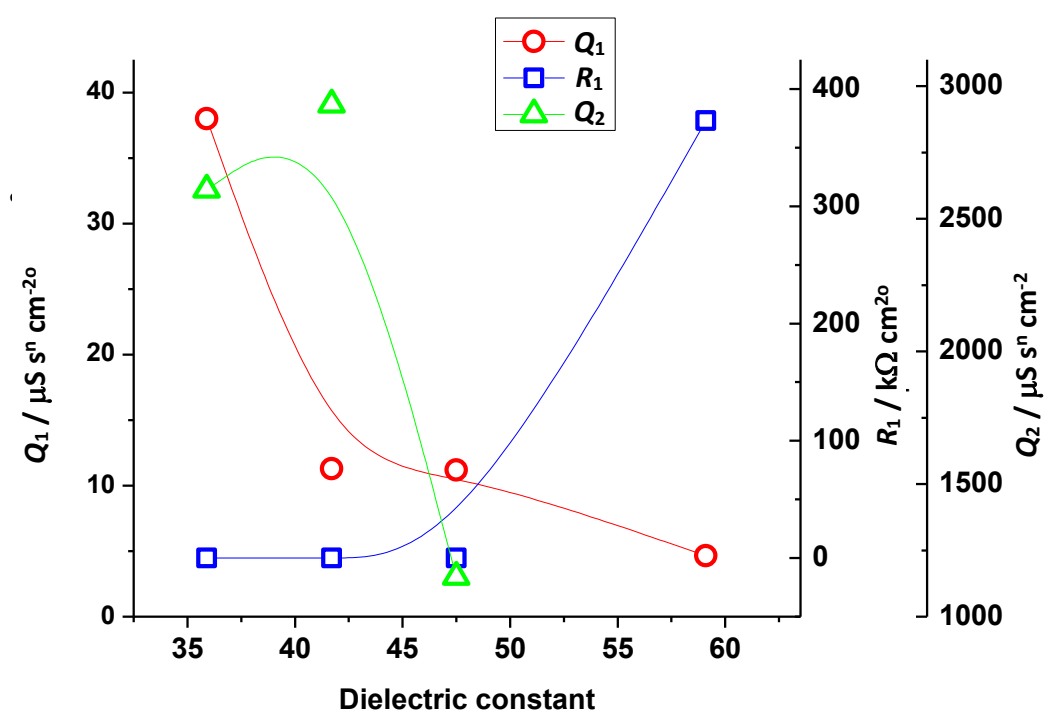

Figure 9. $Q_{1}, Q_{2}$ and $R_{1}$ vs. dielectric constant data of Poly(ProDOT) taken from Table 2

Conducting polymers based on thiophene are used as a transducer in lactose biosensor [45], glucose biosensor [46], vitamin C biosensor [47], and hydrogen peroxide biosensor[48]. The ProDOT can be used as a new material for neural interfaces [49,50], for flexible supercapacitors [51,52], capacitive sensors, and charge storage electrodes.

\section{Conclusions}

In this study, 3,4-propylenedioxythiophene (ProDOT) was successfully electro-coated on SCFME in different electrolytic solutions and solvent mixtures having different dielectric constants. The effect of dielectric properties of solvents on Poly(ProDOT) coating on SFCME was investigated by CV and EIS techniques in detail.

As the solvent dielectric constant increased, the deposition charge density in the system decreased. Accordingly, the highest deposition charge density value was obtained from the coating made in the media with the lowest dielectric constant of 35.9. The deposition charge density of electrogrowth and specific capacitance values versus dielectric constant of solvent shows a similar trend, indicating both processes of electrogrowth and that in monomer free conditions are in line. The highest deposition charge density and specific capacitance were obtained from Poly(ProDOT) synthesized at the lowest solvent dielectric constant medium $(\varepsilon=35.9)$.

All resistance values (charge transfer/transport) are lower, while capacitances (double layer and film) are higher for Poly(ProDOT) films formed in solutions of lower dielectric constant. An opposite trend with high resistance and low capacitances is observed for films formed in solutions of higher dielectric constant where deposition charge densities were decreased.

Increasing the polarity of the solvent increases the solvation of the anion of electrolyte $\left(\mathrm{PF}_{6}^{-}\right)$. In these conditions, increased dipole-dipole interaction between the solvent and dopant $\mathrm{PF}_{6}{ }^{-}$will reduce the mobility of $\mathrm{PF}_{6}{ }^{-}$ions and doping of polymer, resulting in a less conductive material compared to low dielectric conditions.

This study may help researchers pave the way toward more efficient strategies to optimize structural properties of Poly(ProDOT) in different application fields, i.e., flexible electronics, sensors, and organic photovoltaics.

Acknowledgement: The authors acknowledge the suggestions and inputs provided by Dr. Baran Sarac. 


\section{References}

[1] S. Song, G. Xu, B. Wang, J. Gu, H. Wei, Z. Ren, L. Zhang, J. Zhao, Y. Li, Synthetic Metals 278 (2021) 116822. https://doi.org/10.1016/j.synthmet.2021.116822

[2] R. Boguzaite, V. Ratautaite, L. Mikoliunaite, V. Pudzaitis, A. Ramanaviciene, A. Ramanavicius, Journal of Electroanalytical Chemistry 886 (2021) 115132. https://doi.org/10.1016/i.jelechem.2021.115132

[3] G. Prunet, F. Pawula, G. Fleury, E. Cloutet, A.J. Robinson, G. Hadziioannou, A. Pakdel, Materials Today Physics (2021) 100402. https://doi.org/10.1016/j.mtphys.2021.100402

[4] K. Huner, F. Karaman, Materials Research Express 6(1) (2018) 015302. https://doi.org/10.1088/2053-1591/aae462

[5] S. Paul, K. Balasubramanian, Spectrochimica Acta Part A: Molecular and Biomolecular Spectroscopy 245 (2021) 118901. https://doi.org/10.1016/i.saa.2020.118901

[6] K. Yamabe, H. Goto, Fibers and Polymers 19(1) (2018) 248-253. https://doi.org/10.1007/s12221-018-7692-8

[7] B. Lu, S. Zhang, L. Qin, S. Chen, S. Zhen, J. Xu, Electrochimica Acta 106 (2013) 201-208. https://doi.org/10.1016/j.electacta.2013.05.068

[8] D. Zalka, S. Vesztergom, M. Ujvári, G. G. Láng, Journal of Electrochemical Science and Engineering 8(2) (2018) 151-162. https://doi.org/10.5599/jese.508

[9] L. F. Marchesi, S. C. Jacumasso, R. C. Quintanilha, H. Winnischofer, M. Vidotti, Electrochimica Acta 174 (2015) 864-870.https://doi.org/10.1016/i.electacta.2015.05.077

[10] K. Darowicki, J. Kawula, Electrochimica Acta 49(27) (2004) 4829-4839. https://doi.org/10.1016/i.electacta.2004.05.035

[11] K. Cysewska, J. Karczewski, P. Jasiński, Electrochimica Acta 176 (2015) 156-161. https://doi.org/10.1016/j.electacta.2015.07.006

[12] J. Bobacka, A. Lewenstam, A. Ivaska, Journal of Electroanalytical Chemistry 489(1-2) (2000) 17-27. https://doi.org/10.1016/S0022-0728(00)00206-0

[13] A.J. Bard, L.R. Faulkner, Methods 2(482) (2001) 580-632.

[14] X. Cui, D.C. Martin, Sensors and Actuators B: Chemical 89(1-2) (2003) 92-102. https://doi.org/10.1016/S0925-4005(02)00448-3

[15] J. Yang, D.C. Martin, Journal of Materials Research 21(5) (2006) 1124-1132. https://doi.org/10.1557/jmr.2006.0145

[16] S. J. Wilks, S. M. Richardson-Burn, J. L. Hendricks, D. Martin, K. J. Otto, Frontiers in Neuroengineering 2 (2009) 7. https://doi.org/10.3389/neuro.16.007.2009

[17] E. R. Kandel, J. H. Schwartz, T. M. Jessell, S. A. Siegelbaum, A. J. Hudspeth, Principles of Neural Science, Fifth Editon, Cenveo Publisher Services, Columbia, MD, USA, 2013.

[18] S. M. Richardson-Burns, J. L. Hendricks, B. Foster, L. K. Povlich, D.-H. Kim, D. C. Martin, Biomaterials 28(8) (2007) 1539-1552. https://doi.org/10.1016/i.biomaterials.2006.11.026

[19] L. K. Povlich, J. C. Cho, M. K. Leach, J. M. Corey, J. Kim, D. C. Martin, Biochimica et Biophysica Acta (BBA)-General Subjects 1830(9) (2013) 4288-4293. https://doi.org/10.1016/i.bbagen.2012.10.017

[20] X. Strakosas, B. Wei, D. C. Martin, R. M. Owens, Journal of Materials Chemistry B 4(29) (2016) 4952-4968. https://doi.org/10.1039/C6TB00852F

[21] J. M. Murbach, S. Currlin, A. Widener, Y. Tong, S. Chhatre, V. Subramanian, D. C. Martin, B. N. Johnson, K. J. Otto, Mrs Communications 8 (2018) 1043-1049. https://doi.org/10.1557/mrc.2018.138

[22] J. D. Stenger-Smith, C. K. Webber, N. Anderson, A. P. Chafin, K. Zong, J. R. Reynolds, Journal of the Electrochemical Society 149(8) (2002) A973. https://doi.org/10.1149/1.1485773

[23] J. D. Stenger-Smith, A. Guenthner, J. Cash, J. A. Irvin, D. J. Irvin, Journal of the Electrochemical Society 157(3) (2010) A298. https://doi.org/10.1149/1.3276094 
[24] Y. Sulaiman, R. Kataky, Journal of the Electrochemical Society 159(2) (2011) F1. https://doi.org/10.1149/2.019202jes

[25] A. S. Sarac, S. A. Tofail, M. Serantoni, J. Henry, V. J. Cunnane, J. B. McMonagle, Applied Surface Science 222(1-4) (2004) 148-165. https://doi.org/10.1016/i.apsusc.2003.08.008

[26] A. S. Sarac, H.-D. Gilsing, A. Gencturk, B. Schulz, Progress in Organic Coatings 60(4) (2007) 281-286. https://doi.org/10.1016/i.porgcoat.2007.07.025

[27] A. S. Saraç, E. Doğru, M. Ateş, E. A. Parlak, Turkish Journal of Chemistry 30(4) (2006) 401418.

[28] F. G. Guler, A. S. Sarac, Express Polymer Letters 5(6) (2011) 493-505. http://doi.org/10.3144/expresspolymlett.2011.48

[29] P. Yadav, S. Naqvi, A. Patra, RSC Advances 10(21) (2020) 1239512406.https://doi.org/10.1039/DORA01436B

[30] A. Mishra, C.-Q. Ma, P. Bauerle, Chemical Reviews 109(3) (2009) 1141-1276. https://doi.org/10.1021/cr8004229

[31] I.F. Perepichka, D. F. Perepichka, Handbook of Thiophene-Based Materials: Applications in Organic Electronics and Photonics, 2 Volume Set, John Wiley \& Sons, 2009. ISBN: 978-0470-05732-2.

[32] R. Zhang, Y. Huang, L. Liu, Y. Tang, D. Su, L. Xu, Applied Surface Science 257(6) (2011) 18401844. https://doi.org/10.1016/i.apsusc.2010.08.102

[33] T. Karazehir, M. Ates, A. S. Sarac, Journal of the Electrochemical Society 163(8) (2016) G107. https://doi.org/10.1149/2.1011608jes

[34] J. F. Rusling, S. L. Suib, Advanced Materials 6(12) (1994) 922-930. https://doi.org/10.1002/adma.19940061204

[35] N. Elgrishi, K. J. Rountree, B. D. McCarthy, E. S. Rountree, T. T. Eisenhart, J. L. Dempsey, Journal of Chemical Education 95(2) (2018) 197-206. https://doi.org/10.1021/acs.jchemed.7b00361

[36] G. Walter, Corrosion Science 26(9) (1986) 681-703. https://doi.org/10.1016/0010938X(86)90033-8

[37] R. Cottis, S. Turgoose, NACE International, Houston, 2015, TX.77084-4906.

[38] E. Gileadi, Electrode Kinetics for Chemists, Chemical Engineers, and Materials Scientists, Wiley-VCH, Capstone, NY, 1993.

[39] A. J. Bard, M. V. Mirkin (eds.), Scanning Electrochemical Microscopy, CRC Press, 2001. 664. https://doi.org/10.1201/9780203910771

[40] J.R. Macdonald, E. Barsoukov, History 1(8) (2005) 1-13.

[41] T. Girija, M. Sangaranarayanan, Synthetic Metals 156(2-4) (2006) 244-250. https://doi.org/10.1016/i.synthmet.2005.12.006

[42] T. Karazehir, B. Sarac, H.-D. Gilsing, S. Gumrukcu, J. Eckert, A. S. Sarac, Molecular Systems Design \& Engineering 6(3) (2021) 214-233. https://doi.org/10.1039/D0ME00126K

[43] P. Jakhar, M. Shukla, V. Singh, Journal of Materials Science: Materials in Electronics 30(4) (2019) 3563-3573. https://doi.org/10.1007/s10854-018-00634-w

[44] P. Jakhar, M. Shukla, V. Singh, Journal of the Electrochemical Society 165(7) (2018) G80. https://doi.org/10.1149/2.0461807jes

[45] V. Safarnavadeh, K. Zare, A. R. Fakhari, Biosensors and Bioelectronics 49 (2013) 159-163. https://doi.org/10.1016/i.bios.2013.04.043

[46] M. Şenel, M. Dervisevic, E. Çevik, Current Applied Physics 13(7) (2013) 1199-1204. https://doi.org/10.1016/i.cap.2013.03.004.

[47] Y. Wen, X. Duan, J. Xu, R. Yue, D. Li, M. Liu, L. Lu, H. He, Journal of Solid State Electrochemistry 16(12) (2012) 3725-3738. https://doi.org/10.1007/s10008-012-1803-7 
[48] D. Li, Y.-p. Wen, J.-k. Xu, H.-h. He, M. Liu, Chinese Journal of Polymer Science 30(5) (2012) 705-718. https://doi.org/10.1007/s10118-012-1167-6

[49] M. Asplund, T. Nyberg, O. Inganäs, Polymer Chemistry 1(9) (2010) 1374-1391. https://doi.org/10.1039/COPY00077A

[50] U. A. Aregueta-Robles, A. J. Woolley, L. A. Poole-Warren, N. H. Lovell, R. A. Green, Frontiers in Neuroengineering 7 (2014) 15. https://doi.org/10.3389/fneng.2014.00015

[51] S. Palchoudhury, K. Ramasamy, R. K. Gupta, A. Gupta, Frontiers in Materials 5 (2019) 83. https://doi.org/10.3389/fmats.2018.00083

[52] Y. Han, L. Dai, Macromolecular Chemistry and Physics 220(3) (2019) 1800355. https://doi.org/10.1002/macp.201800355 